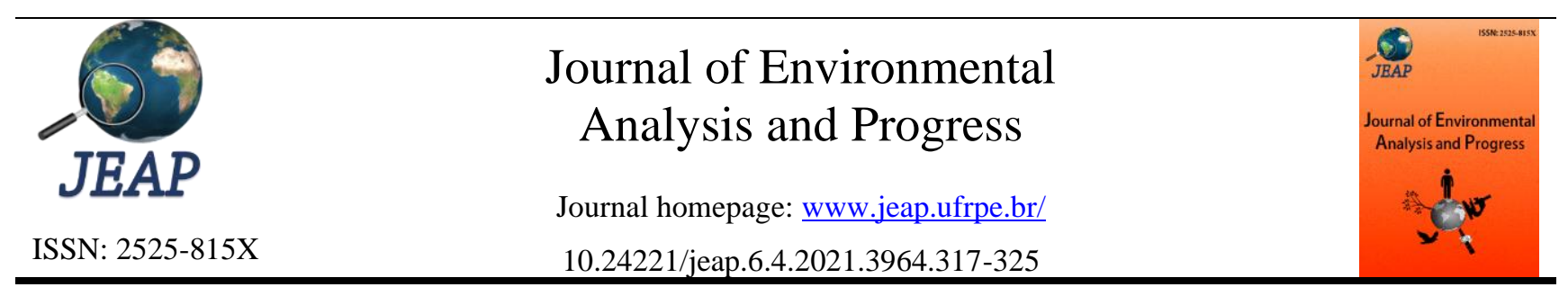

\title{
Incidência de acidentes ofídicos na microrregião de Birigui-SP
}

\section{Incidence of snakebites in the microregion of Birigui-SP}

\author{
Lucinéia Correa de Albuquerque Souza ${ }^{\mathrm{a}}$, Aparecida de Fátima Michelin ${ }^{\mathrm{a}}$ \\ ${ }^{a}$ Universidade Paulista-UNIP, Campus Araçatuba. Avenida Baguaçu, n. 1939, Jardim Alvorada, Araçatuba, São Paulo, \\ Brasil. CEP: 16018-555. E-mail: lucineiacorreaalbuquerque@ gmail.com, cidinhamichelin@gmail.com.
}

A R T I C L E I N F O

Recebido 23 Nov 2020

Aceito 04 Mai 2021

Publicado 13 Out 2021

\begin{abstract}
A B S T R A C T
Snakebite accidents are considered the most complex among those caused by venomous animals, due to their specificity, with variations in clinical, prognostic, and immunological characteristics. The present research aimed to verify the frequency of reported snake accidents and characterize the accident, the snake, the serotherapy used, and the injured person, including its outcome, in the microregion of Birigui-SP. Data collection, duly approved by the Research Ethics Committee, was carried out in the Individual Notification Forms of the Diseases Information and Notification System of the Center for Epidemiological Surveillance in the city of Birigui-SP. Based on the verified records carried out from 2010 to 2018, there were 35 notifications of snakebites. The majority were of the Bothrops (Jararaca) (49\%) and Crotalus (Cascavel) (17\%), while the minority was of the elapid type (Coral) $3 \%$ and non-venomous $(6 \%)$, and $26 \%$ of the notifications the snake gender was ignored. Such accidents mainly affected men (63\%), adults aged up to 60 years old $(69 \%)$ and occurred in rural areas $(71 \%)$. As for the location of the body, $74 \%$ of accidents affected the extremities of the upper and lower limbs. All victims were healed, and most of them reacted with local or systemic manifestations. Although all victims had a good evolution, it is important to emphasize the importance of preventing such occurrences, especially in rural and urban environments, especially in men, emphasizing the upper and lower limbs, the sites most affected by snakes of the Bothrops and Crotalus genus.
\end{abstract}

Keywords: Ophidian accident, passive immunization, snakebite.

\section{R E S U M O}

Os acidentes por picadas de serpentes são considerados os mais complexos dentre aqueles causados por animais peçonhentos, devido à sua especificidade, com variações quanto às características clínicas, prognósticas e imunológicas. A presente pesquisa objetivou verificar a frequência dos acidentes ofídicos notificados e caracterizar o acidente, a serpente, a soroterapia empregada e o acidentado, inclusive o seu desfecho, na microrregião de Birigui-SP. A coleta de dados, devidamente aprovada pelo Comitê de Ética e Pesquisa, foi realizada nas Fichas de Notificação Individual do Sistema de Informação e Notificação de Agravos do Centro de Vigilância Epidemiológica do município de Birigui-SP. Com base nos registros verificados, realizados no período de 2010 a 2018, ocorreram 35 notificações de acidentes ofídicos, sendo que a maioria foi do tipo botrópico (Jararaca) (49\%) e crotálico (Cascavel) (17\%), enquanto que a minoria, do tipo elápidico (Coral) $3 \%$ e não peçonhento (6\%), e em $26 \%$ das notificações o gênero da serpente foi ignorado. Tais acidentes atingiram principalmente homens (63\%), adultos com até 60 anos $(69 \%)$ e ocorreram na zona rural $(71 \%)$. Quanto ao local do corpo, $74 \%$ dos acidentes atingiram as extremidades dos membros superiores e inferiores. Todos os acidentados tiveram cura, sendo que a maioria apresentou algum tipo de reação com manifestação local e ou sistêmica. Embora todos os acidentados tenham tido uma boa evolução, é importante ressaltar a importância da prevenção de tais ocorrências, tanto no ambiente rural, quanto no urbano, 
especialmente os homens, com destaque para os membros superiores e inferiores, os locais mais atingidos pelas serpentes do gênero Bothrops e Crotalus.

Palavras-Chave: Acidente ofídico, imunização passiva, picada de serpentes.

\section{Introdução}

Os animais peçonhentos são aqueles que possuem glândulas produtoras e secretoras de veneno que é composto por uma mistura complexa de substâncias (França \& Málaque, 2009) enzimáticas, peptídicas e por toxinas que ao ser inoculado em um organismo possui a capacidade de induzir resposta imunológica (Cunha \& Martins, 2012) pró inflamatória (Carvalho et al., 2019).

Dentre os acidentes com animais peçonhentos, os ofídicos que são aqueles causados por picada de serpentes ocorrem em toda a extensão global e acometem, anualmente, cerca de 2,7 milhões de indivíduos. Atingem especialmente as populações de países tropicais, em desenvolvimento populacional e com desigualdade de acesso à saúde pública (Williams et al., 2019).

Vital Brazil, em seu primeiro estudo sobre o ofidismo, ocorrido em 1901, considerou os acidentes com serpentes como um problema de saúde pública, com maior incidência no interior do Brasil, envolvendo principalmente os trabalhadores em áreas agrícolas (Brasil, 1901).

Para promover ações de controle dos acidentes por animais peçonhentos, a antiga Secretaria Nacional de Ações Básicas em Saúde do Ministério da Saúde (SNABS/MS) tornou obrigatório, a partir de 1986, o registro de todos os casos, devido à crise na produção do soro, conforme relatado por Bochner \& Struchiner, (2003).

Esse agravo foi incluído na Lista de Notificação Compulsória (LNC), de acordo com a Portaria $\mathrm{n}^{\mathrm{o}} 2.472$, de 31 de agosto de 2010. O registro inicia-se pelo preenchimento da Ficha Individual de Notificação (FIN) que contém todos os dados do acidentado e perfil da serpente, bem como as manifestações clínicas, sorologia empregada e o desfecho. Posteriormente, a FIN é encaminhada para a Vigilância Epidemiológica do município e repassada para a Secretaria Estadual de Saúde que alimenta o Sistema de Informação de Agravos de Notificação (SINAN), o qual mantém a regularidade informativa e investigativa dos casos de doenças e agravos de notificação compulsória do Ministério da Saúde (SINAN, 2019).

O Brasil, junto com o Vietnã e atrás apenas da Índia e Sri Lanka, ocupa o terceiro lugar na taxa de incidência de envenenamento por serpentes (Kasturiratne et al., 2008). As diversidades regionais de acessos e cuidados adequados à saúde, tornou esse tipo de ocorrência um problema sanitário e desse modo foi incluído como uma das Doenças Tropicais Negligenciadas (DTN) (Chiepaux, 2017).

Os acidentes ofídicos causados por serpentes peçonhentas e notificados no Brasil são classificados em botrópico (serpente Jararaca); crotálico (serpente Cascavel); laquético (serpente Surucucu) e elapídico (serpente Coral). Dentre estes, os acidentes botrópicos constituem em $90 \%$ dos acidentes ofídicos anuais ocorridos no Brasil (Melgarejo, 2009), por se tratar da espécie de serpente mais abundante na região Sul da América (Bochner \& Struchiner, 2003).

As manifestações clínicas e reacionais dos acidentados com picadas por serpentes peçonhentas variam entre as espécies envolvidas e podem ser resultantes de ações proteolíticas, hemorrágicas, coagulantes, neurotóxicas e miotóxicas (Bernarde \& Gomes, 2012; Melgarejo, 2009). Nos acidentes botrópicos, o veneno inoculado possui ação proteolítica, coagulante e hemorrágica (França \& Málaque, 2009); nos acidentes crotálicos, o veneno apresenta ação neurotóxica, miotóxica, coagulante e hepatotóxica (Barraviera et al., 1989; Barraviera et al., 1995); em acidentes laquéticos, a ação do veneno é proteolítica, hemorrágica, coagulante e possivelmente neurotóxica (Souza, 2009). Os acidentes elapídicos, que ocasionam apenas $1 \%$ de letalidade, a principal atividade do veneno é neurotóxica (Bernarde \& Gomes, 2012; Melgarejo, 2009).

Assim sendo, pode-se considerar que o quadro clínico do acidentado está mais frequentemente relacionado com a ação local e sistêmica do veneno, e mais raramente às reações de hipersensibilidade a essa toxina. As complicações mais frequentes estão relacionadas aos sistemas cardiovascular, renal e respiratório. A redução no número de casos complicados, possivelmente, tem relação com a melhor qualidade do atendimento prestado ao acidentado e com a disponibilidade da imunoterapia (Amaral, 2009).

A principal medida de ação médica é o diagnóstico correto para administrar o soro antiofídico específico (Pinho \& Pereira, 2001) e resultar em uma terapia eficiente (Bochner \& Struchiner, 2003). Para tal ação, deve-se levar em consideração o porte e a espécie de serpente, o local e a quantidade do veneno inoculado, o 
tempo percorrido entre o momento do acidente e o início da soroterapia (Jorge \& Ribeiro, 1997), além das alterações hemodinâmicas e reacionais resultantes do efeito tóxico da peçonha (Sitprija \& Sitprija, 2012). Após a administração do soro, o efeito neutralizante da toxina será imediato (Araujo et al., 2008). Assim, a injeção intravenosa do concentrado de anticorpos heterólogos, mais especificamente, imunoglobulina $\mathrm{G}$ ( $\mathrm{IgG}$ ) ou porção $\mathrm{Fab}^{2}$ da $\mathrm{IgG}$, promoverá formação de complexos neutralizantes do veneno que se encontra disperso no plasma do paciente, resultando na prevenção de sequelas (Sanhajariya, Duffull \& Isbister, 2018). Todavia, a soroterapia é ineficaz para os efeitos locais e pode provocar reações adversas, como a hipersensibilidade do tipo I em cerca de $20 \%$ dos casos, e, com menor frequência, a reação de hipersensibilidade tipo III, dependendo da resposta imune do indivíduo (Wen, 2009). Cabe ressaltar que o efeito neutralizante da toxina e a dose administrada se baseiam nas pesquisas in vitro e em animais que, por sua vez, são diferentes, comparado aos efeitos em humanos (Maduwage, 2016). O soro antibotrópico produzido pelo Instituto Butantan é capaz de inibir efetivamente a atividade da metalopeptidase e de algumas das serinas peptidases, as principais toxinas presentes no veneno da Bothrops jararaca. As atividades destas peptidases podem estar relacionadas com algumas das amplas manifestações nocivas decorrentes do envenenamento por essa espécie de serpente (Kuniyoshi et al., 2012; Kuniyoshi et al., 2017).

A produção do soro antiofídico no Brasil está a cargo do Instituto Butantan, Instituto Vital Brasil, Fundação Ezequiel Dias e Centro de Produção e Pesquisa de Imunobiológicos que utilizam os cavalos para a inoculação do veneno da serpente a fim de promover estimulação e resposta imunológica do animal para produzir anticorpos heterólogos (Salomão, Oliveira-Luna \& Machado, 2018). A partir do plasma (Sanhajariya, Duffull \& Isbister, 2018), o soro será separado e qualificado para o direcionamento aos locais referenciados para a sua utilização (Cardoso, Yamaguchi \& Moura-da-Silva, 2009), conforme os dados epidemiológicos notificados (SINAN, 2019).

$\mathrm{O}$ uso racional dos antivenenos e a disponibilização nos locais de acesso constituem a estratégia mais eficaz para tratar e reduzir o tempo entre o acidente e os cuidados terapêuticos. Desta forma, vincular os dados epidemiológicos notificados dos acidentes ofídicos registrados no SINAN são fundamentais para planejar e orientar as estratégias de produção e distribuição dos soros antivenenos, segundo os pontos de atendimento médico dos acidentados, definidos pelas Secretarias Estaduais de Saúde (SVS $\left.{ }^{1}, 2019\right)$, conforme os riscos de cada área geográfica (Matos \& Ignotti, 2020).

$\mathrm{O}$ estudo objetivou determinar a frequência de acidentes ofídicos notificados, bem como, verificar as informações relativas ao acidentado, ao acidente e à serpente.

\section{Material e Métodos}

Caracterização da área de estudo

A microrregião de Birigui está localizada na região Noroeste do estado de São Paulo, com bioma de Mata Atlântica e produção agrícola com lavouras temporárias e permanentes, predominando a cultura de cana-de-açúcar (Silva et al., 2012; IBGE, 2019). É composta pelos municípios de Birigui, município sede, (Latitude: $21^{\circ} 17^{\prime} 21^{\prime \prime}$ Sul, Longitude: 50²0'28" Oeste), Coroados (Latitude: 21 21'32" Sul, Longitude: 50 17'22" Oeste), e Santópolis do Aguapeí (Latitude: 21³8'19" Sul, Longitude: 50³0'19" Oeste), totalizando uma população estimada de 135.829 pessoas (IBGE, 2020).

\section{Base de dados}

Após a aprovação do estudo pelo Comitê de Ética e Pesquisa da Universidade Paulista UNIP, CAAE 03499418.0.0000.5512 e protocolo 3.087.235, foi realizada a coleta dos dados no Centro de Vigilância Epidemiológica do município de Birigui-SP, por meio das Fichas do Sistema de Informação e Notificação de Agravos (SINAN), referentes ao período de 2010 a 2018.

As variáveis utilizadas foram: notificação individual (sexo, idade); antecedentes epidemiológicos (data do acidente, zona de ocorrência, tempo decorrido da picada, local da picada); dados clínicos ( manifestações locais e manifestações sistêmicas); dados do acidente (gênero da serpente); tratamento; complicações locais e sistêmicas e evolução do caso.

\section{Análise estatística}

Os dados coletados foram dispostos em delineamento descritivo e exploratório, e seus resultados foram apresentados em valores absolutos e/ou relativos após tabulados e calculados.

\section{Resultados}

Conforme os dados epidemiológicos disponibilizados pelo Sistema de Informação de Agravos de Notificação (SINAN), foram realizadas 35 notificações de acidentes ofídicos no período de 2010 a 2018 (Figura 1). 


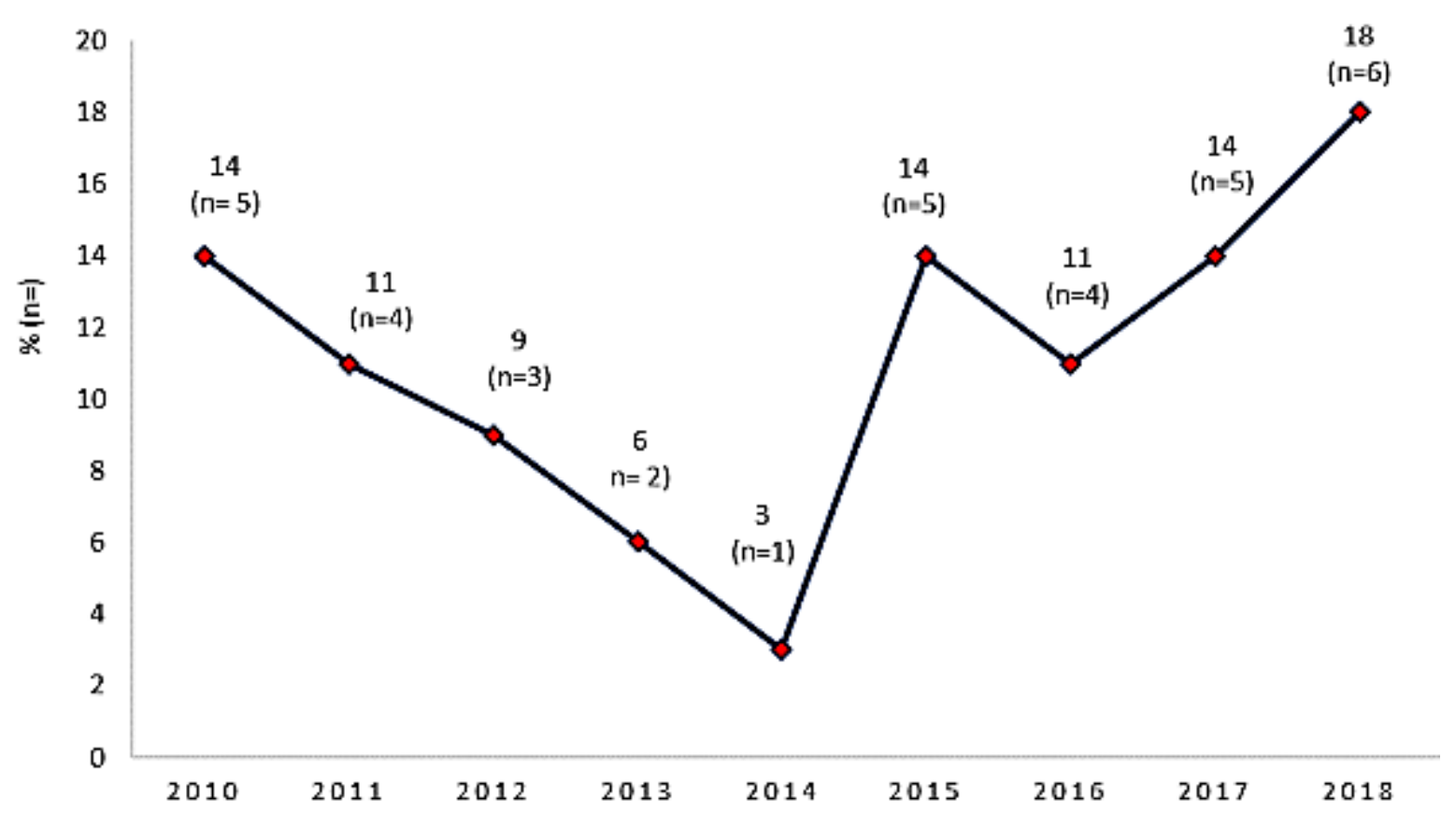

Figura 1. Distribuição absoluta e relativa de notificações de acidentes ofídicos no período de 2010 a 2018. Microrregião de Birigui-SP. Fonte: Centro de Vigilância Epidemiológica de Birigui-SP. Fonte: Adaptado de Centro de Vigilância Epidemiológica de Birigui-SP (2019).

No que diz respeito à notificação individual, a maior frequência de casos de acidentes ofídicos ocorreu com indivíduos do sexo masculino com 28 casos $(80 \%)$ e adultos (de 18 a 60 anos) com 24 casos (69\%), seguido de idosos (acima de 60 anos) com 8 casos (23\%) e 3 casos (9\%) de jovens (menores de 18 anos). Em relação aos antecedentes epidemiológicos, 63\% (22) dos casos ocorreu na zona rural, 29\% (10) na zona urbana, 3\% (1) em zona periurbana e 6\% (2) deles sem registro do local. O tempo, em horas, entre o momento da picada e o local de atendimento, ocorreu, em sua maioria, na primeira hora (0 a 1 hora), com $63 \%$ (22) dos casos, seguido de $14 \%$ (5) dos casos, entre 1 e 3 horas e, os demais, com $17 \%$ (6) acima deste intervalo de tempo, chegando a superar 24 horas, e para dois casos, o tempo não foi informado. No que se refere ao local do corpo em que ocorreu a picada, 73\% (26) dos acidentes acometeu as extremidades de membros superiores e inferiores (Tabela 1).

Tabela 1. Distribuição dos acidentes ofídicos, segundo notificação individual (sexo e faixa etária) e antecedentes epidemiológicos (zona, tempo percorrido para o atendimento e local da picada), notificados no período de 2010 a 2018. Microrregião de Birigui-SP. Fonte: Centro de Vigilância Epidemiológica de Birigui-SP (2019).

\begin{tabular}{lcc}
\hline Variáveis & n & \% \\
\hline Sexo & & \\
Masculino & 28 & 80 \\
\hline
\end{tabular}

\begin{tabular}{lll}
\hline Feminino & 7 & 20 \\
Faixa etária & & \\
Jovens < 18 anos & 3 & 9 \\
Adultos até 60 anos & 24 & 69 \\
Idosos 60 anos & 8 & 23 \\
Zona & & \\
Rural & 22 & 63 \\
Urbana & 10 & 29 \\
Periurbana & 1 & 3 \\
Ignorado & 2 & 6 \\
Tempo percorrido para o atendimento & & \\
0 a 1 hora & 22 & 63 \\
1 a 3 horas & 5 & 14 \\
Acima de 3 horas & 6 & 17 \\
Ignorado & 2 & 6 \\
Local da picada & & \\
Antebraço & 2 & 6 \\
Mão & 8 & 23 \\
Dedo da mão & 5 & 14 \\
Perna & 7 & 20 \\
Pé & 8 & 23 \\
Dedo do pé & 5 & 14 \\
\hline
\end{tabular}

Considerando os dados clínicos, as ocorrências foram distribuídas em manifestações locais $89 \%$ (31), com 3\% (1) ignorado e sistêmicas $6 \%$ (2), com 6\% (2) ignorado. Os sintomas locais da dor $91 \%$ (32) ocorreram, na maioria dos casos, seguidos por edema 54\% (19) e, a minoria, com equimose $6 \%$ (2).

Em apenas 1 caso (3\%) foi descrito eritema no campo denominado "outros". Quanto 
às manifestações clínicas sistêmicas, ocorreu reação miolítica/hemolítica em 1 (3\%) caso. Em apenas 1 caso (3\%) foi descrito sudorese e malestar no campo denominado "outros" (Tabela 2).

Tabela 2. Distribuição dos acidentes ofídicos, segundo dados clínicos (manifestações locais e sistêmicas), notificados no período de 2010 a 2018, na Microrregião de Birigui-SP. Fonte: Adaptado do Centro de Vigilância Epidemiológica de Birigui-SP (2019).

\begin{tabular}{lll}
\hline Variáveis & $\mathbf{n}$ & $\mathbf{\%}$ \\
\hline Manifestação local & & \\
Dor & 31 & 89 \\
Edema & 20 & 57 \\
Equimose & 2 & 6 \\
Outros (eritema) & 1 & 3 \\
Ignorado & 3 & 9 \\
Manifestação sistêmica & & \\
Mal-estar e sudorese & 1 & 3 \\
Miolítica/hemolítica (anemia, mialgia, urina escura) & 1 & 3 \\
\hline
\end{tabular}

Em relação aos dados do acidente ofídico, predominaram o tipo botrópico (Jararaca) $49 \%$ (17) e crotálico (Cascavel) 17\% (6) e houve menor prevalência do tipo elapídico (Coral) (1) $3 \%$. Dois (6\%) acidentes com serpentes não peçonhentas foram relatados. Deve-se destacar que o tipo de acidente foi relatado como ignorado em 26\% (9) dos acidentes (Tabela 3).

Tabela 3. Distribuição dos acidentes ofídicos, segundo dados dos acidentes ofídicos, notificados no período de 2010 a 2018, na Microrregião de Birigui-SP. Fonte: Adaptado do Centro de Vigilância Epidemiológica de Birigui-SP (2019).

\begin{tabular}{lll}
\hline Variáveis & n & \% \\
\hline Tipo de acidente & & \\
Botrópico & 17 & 49 \\
Crotálico & 6 & 17 \\
Elapídico & 1 & 3 \\
Não peçonhenta & 2 & 6 \\
Ignorada & 9 & 26 \\
\hline
\end{tabular}

Quanto ao tratamento com soro antiofídico, a maioria fez uso, ou seja, 57\% (20), enquanto $29 \%$ (10) não o utilizaram e $14 \%$ (5) foi ignorado. Em relação à gravidade do acidente, 43\% (15 casos) foram classificados como leves, $37 \%$ (13 casos) como moderados e 9\% (3 casos) como graves. Em 4 casos (11\%) não foi registrada a gravidade do acidente. Dos 35 acidentes ofídicos registrados, 97\% (34) dos casos evoluíram para a cura e 1 caso (3\%) foi ignorado (Tabela 4).

Tabela 4. Distribuição dos acidentes ofídicos, segundo o tratamento (sorologia, complicação e gravidade) e evolução dos casos, notificados no período de 2010 a 2018, Microrregião de BiriguiSP. Fonte: Adaptado do Centro de Vigilância Epidemiológica de Birigui-SP (2019).

\begin{tabular}{lll}
\hline Variáveis & n & \% \\
\hline Sorologia & & \\
$\quad$ Usou soro & 20 & 57 \\
Não usou soro & 10 & 29 \\
Ignorado & 5 & 14 \\
Complicação local & & \\
Déficit funcional & 1 & 3 \\
Grau de gravidade & & \\
$\quad$ Leve & 15 & 43 \\
Moderado & 13 & 37 \\
$\quad$ Grave & 3 & 9 \\
Ignorado & 4 & 11 \\
Evolução & & \\
$\quad$ Cura & 34 & 97 \\
Ignorada & 1 & 3 \\
\hline
\end{tabular}

\section{Discussão}

Os acidentes com serpentes atingiram, em sua maioria, os indivíduos do sexo masculino (80\%) e adultos de até 60 anos (69\%), especialmente em área rural. No Brasil, a faixa etária que apresenta maior incidência é a de 40 a 59 anos, com 17,1 \% dos casos (Machado, 2018). Tais acidentes ocorreram, principalmente, em áreas rurais (63\%), mas é importante destacar a expressiva ocorrência em zona urbana (29\%). Neste caso, podem estar relacionados à adaptabilidade das serpentes ao ambiente urbano, (Silva, 2019) motivadas pela destruição de florestas, devido à expansão agropecuária (Navega-Gonçalves \& Porto, 2016). A substituição da vegetação por lavoura causa uma evidente modificação da fauna de répteis. No caso da Bothrops jararaca, existe a possibilidade de ser beneficiada devido ao aumento de luminosidade e favorecimento da presença de roedores, principal presa para a serpente adulta (Marques et al., 2004).

Os acidentes acometeram, principalmente os membros inferiores e superiores, nos pés e 
mãos (23\%), no dedo do pé e mão, também com igual frequência (14\%), e na perna (20\%). Assim sendo, a proteção das partes do corpo expostas ao risco é de grande importância na prevenção dos acidentes ofídicos (Pinho \& Pereira, 2001). Neste contexto, recomenda-se o uso de equipamentos de proteção individual (EPIs), como botas e luvas, adequados para a referida exposição. Tal perfil de acidentes pode estar relacionado às peculiaridades de trabalho dos homens e às atividades de lazer. Segundo Silva (2019), as serpentes são encontradas mais facilmente em períodos quentes, devido ao fato de hibernarem no inverno e saírem no verão para se alimentar. Desta forma, adultos envolvidos com atividades rurais, seja a trabalho em área agrícola, manutenção de terrenos, fazendas e residências, quanto crianças e jovens em situações de férias em atividades ao ar livre, acabam contribuindo para a ocorrência de acidentes envolvendo serpentes.

As serpentes do gênero Bothrops foram responsáveis por $49 \%$ dos acidentes na microrregião de Birigui-SP. No Brasil, encontrase a grande maioria das espécies desse gênero, sendo que a espécie $B$. jararaca habita desde o sul da Bahia até o rio Grande do Sul, sendo a espécie mais encontrada na região Sudeste (Melgarejo, 2009). Também é importante ressaltar a influência o comportamento antrópico, uma vez que facilita a aproximação do homem com esses animais (Matos \& Ignotti, 2020).

A maioria dos acidentados (77\%) receberam recebeu atendimento rápido, ou seja, nas três primeiras horas. $\mathrm{O}$ atendimento rápido é essencial para reduzir a forma grave ou a evolução da morbimortalidade. Nas regiões Sul e Sudeste do Brasil, no período de 2001 a 2012, a maioria dos jovens acidentados foi atendida em igual espaço de tempo. Certamente, a presteza no atendimento é um reflexo da estruturação da rede de saúde para o desfecho adequado desses acidentes (Bochner, Fiszon \& Machado, 2014).

Dentre os três casos registrados como grave, um foi detalhado para melhor caracterizar a sua evolução, cuja vítima do sexo masculino, e com idade de 26 anos, sofreu o acidente na região periurbana, durante o exercício do seu trabalho. $\mathrm{O}$ atendimento médico ocorreu após 24 horas da ocorrência do acidente, sem identificação, nem mesmo presunção do tipo de serpente. As manifestações clínicas relatadas foram: dor no local da picada, edema, sudorese e mal-estar. O tratamento foi realizado com soro heterólogo específico e o caso evoluiu para a cura do acidentado. Cabe ressaltar que, além da importância do tempo transcorrido entre o acidente e o atendimento médico, identificar o tipo de serpente é fundamental para o diagnóstico e a prescrição de soroterapia específica, contribuindo para o uso racional do antiveneno (Wen, 2009).

Os acidentes que ocorrem em locais com difícil acesso ao tratamento, como na Amazônia brasileira, tendem a ter o início do tratamento com $\mathrm{o}$ antiveneno retardado, às vezes, com subdosagens, ou mesmo não realizado, contribuindo para o desfecho com quadros clínicos mais graves, sequelas permanentes e mortes (Feitosa et al., 2015; Wen et al., 2015). Em contrapartida, também há o relato de uso excessivo de antiveneno, que contribui para o desfecho negativo desses acidentes (Santana \& Oliveira, 2020).

Além dos acidentados graves (9\%), todos os demais foram submetidos à soroterapia, exceto dois casos, dentre os moderados. O Ministério da Saúde preconiza normas para a realização de soroterapia, conforme classificação da gravidade do acidente, que é feita a partir das manifestações clínicas locais e sistêmicas. Assim sendo, um acidente botrópico moderado deve ser tratado com soro antibotrópico específico e, quando não for possível, recomenda-se utilizar a combinação de soros, sempre seguido das observações e reavaliações contínuas do acidentado, para evitar complicações e, se necessário, fazer a reclassificação do caso $\left(\mathrm{SVS}^{2}, 2019\right)$.

Embora não tenha ocorrido nenhum registro de óbito, merecem ser destacadas as manifestações locais (89\%) e sistêmicas (6\%) que podem comprometer a evolução clínica e levar a um desfecho fora do controle. Cabe lembrar que informações sobre o pós-desfecho dos acidentados, no tocante à presença de sequelas, não foram possíveis de serem obtidas, uma vez que os campos a serem preenchidos com tais informações, segundo Machado (2018), foram retirados das fichas de notificação por acidentes peçonhentos.

Por fim, para a maioria das variáveis analisadas, o campo "ignorado" ocorreu, com frequência, em todos os casos, e tal fato é preocupante. A Organização Mundial de Saúde afirma que dados notificados e registrados de formas ineficazes causam danos nos parâmetros utilizados para o direcionamento dos planos de ações e soluções nas estratégias epidemiológicas de melhorias frente aos acidentes ofídicos (WHO, 2020). Segundo o Boletim Epidemiológico da Secretaria de Vigilância em Saúde do Ministério da Saúde (2019), é de extrema importância desenvolver boas estratégias para obter registros de notificações mais rigorosos, com qualidade das informações registradas, a fim de resultar em 
parâmetros mais seguros, confiáveis e realista dos problemas notificados. Portanto, a falta dessa informação impediu maior avanço na interpretação dos resultados obtidos nesse estudo.

\section{Conclusão}

A maioria dos acidentes ofídicos notificados foi causada por serpentes do gênero Bothrops em trabalhadores do sexo masculino, com até 60 anos, principalmente na zona rural. A imunoterapia se mostrou indicada para a minimização de óbitos por pessoas atacadas por ofídios deste gênero.

\section{Agradecimentos}

Os autores agradecem ao Centro de Vigilância Epidemiológica de Birigui/SP.

\section{Referências}

Amaral, F. S. A. 2009. Cuidados intensivos nos acidentes por animais peçonhentos. In: Cardoso, J. L. C.; França, F. O. S., Wen, F. H.; Málaque, C. M. S.; Haddad Jr., V. Animais Peçonhentos no Brasil: Biologia, clínica e terapêutica dos acidentes. 2 ed., Savier, pp. 446-453.

Araujo, H. P.; Bourguignon, S. C.; Boller, M. A. A.; Dias, A. A. S. O.; Lucas, E. P. R; Santos, I. C.; Delgado, I. F. 2008. Potency evaluation of antivenoms in Brazil: The national control laboratory experience between 2000 and 2006. Toxicon, 51, (4), 502-514.

Barraviera, B.; Bonjorno Junior, J. C.; Arakaki, D.; Domingues, M. A. C.; Pereira, P. C.M.; Mendes, R. P.; Machado, J. M.; Meira, D. A. 1989. A retrospective study of 40 victims of Crotalus snake bites. Analysis of the hepatic necrosis observed in one patient. Rev. Soc. Bras. Med. Trop., 22, (1), 5-12.

Barraviera, B.; Coelho, K. Y. R.; Curi, P. R.; Meira, D. A. 1995. Liver disfunction in patients bitten by Crotalus durissus terrificus (Laurenti, 1768) snakes in Botucatu (State of São Paulo, Brazil). Rev. Inst. Med. Trop. São Paulo, 37, (1), 63-69.

Bernarde, P. S.; Gomes, J. O. 2012. Serpentes peçonhentas e ofidismo em Cruzeiro do Sul, Alto Juruá, Estado do Acre, Brasil. Acta Amaz., 42, (1), 65-72.

Bochner, R.; Struchiner, C. J. 2003. Epidemiologia dos acidentes ofídicos nos últimos 100 anos no Brasil: uma revisão. Cad. Saúde pública, 19, (1), 7-16.

Bochner, R.; Fiszon, J. T.; Machado, C. 2014. A Profile of Snake Bites in Brazil, 2001 to 2012. J. Clin. Toxicol., 4, (3), 1-7.
Brasil, V. 1901. Contribuição ao estudo do veneno ophidico. Rev. Med. S. Paulo, IV, 255-260.

Cardoso, D. F.; Yamaguchi, I. K.; Silva, A. M. 2009. Produção de soros antitoxinas e perspectivas de modernização por técnicas de biologia molecular. In: Cardoso, J. L. C.; França, F. O. S., Wen, F. H.; Málaque, C. M. S.; Haddad Jr., V. Animais Peçonhentos no Brasil: Biologia, clínica e terapêutica dos acidentes. 2 ed., Savier, pp. 419-431.

Carvallho, A. E. Z.; Giannotti, K.; Leiguez Jr., E.; Matsubara, M.; Santos, M. C.; Fortes-Dias, C. L.; Teixeira, C. 2019. Crotalus durissusruruima snake venomand a phospholipase A2 isolated from this venom elicit macrophages to form lipid droplets and synthesize inflammatory lipid mediators. J. Immunol. Res., 2019, 2745286.

Chippaux J. P. 2017. Snakebite envenomation turns again into a neglected tropical disease! Venom. Anim. Toxins. Incl. Trop. Dis., 23, 38.

Cunha, E. M.; Martins, O. A. 2012. Principais compostos químicos presentes nos venenos de cobras dos gêneros Bothrops e Crotalus uma revisão. REEC, 2, (2), 21-26.

França, F. S.; Málaque, C. M. S. 2009. Acidente botrópico. In: Cardoso, J. L. C.; França, F. O. S.; Wen, F. A; Málaque, C. M. S; Haddad Jr., V. Animais Peçonhentos no Brasil: biologia, clínica e terapêutica dos acidentes, 2 ed., Savier, pp. 81-95.

Feitosa, E. L.; Sampaio, V. S.; Salina, J. L.; Queiroz, A. M.; Silva, I. M.; Gomes, A. A.; Sachett, J.; Siqueira, A. M.; Ferreira, L. C. L.; Santos, M. C.; Lacerda, M.; Monteiro, W. 2015. Older age and time to medical assistance are associated with severity and mortality of snakebites in the Brazilian Amazon: a case-control study. PLoS ONE, 1-15.

IBGE. 2019. Instituto Brasileiro de Geografia e Estatística. Disponível em: https://cidades.ibge.gov.br/ Acesso em: $12 / 2020$.

IBGE. 2020. Instituto Brasileiro de Geografia e Estatística. Disponível em: https://cidades.ibge.gov.br/ Acesso em: 12/2020.

Jorge, M. T.; Ribeiro, L. A. 1997. Dose de soro (antiveneno) no tratamento do envenenamento por serpentes peçonhentas do gênero Bothrops. Rev. Assoc. Med. Bras., 43, (1), 74-7.

Kasturiratne A.; Wickremasinghe, A. R.; Silva, N.; Gunawardena, N. K.; Pathmeswaran, A.; Premaratna R.; Savioli, L.; Lalloo, D. G.; 
Silva, H. J. 2008. The Global Burden of Snakebite: A Literature Analysis and Modelling Based on Regional Estimates of Envenoming and Deaths. PLoSMed, 5, (11), 1591-1604.

Kuniyoshi, A. K.; Rocha, M.; Carvalho, D. C.; Juliano, M. A.; Juliano Neto, L.; Tambourgi, D. V.; Portaro, F. C. V. 2012. Angiotensindegrading serine peptidase: A new chymotrypsin-like activity in the venom Bothrops jararaca partially blocked by the commercial antivenom. Toxicon, 59, 124131.

Kuniyoshi, A. K.; Kodama, R. T.; Moraes, L. H. F.; Duzzi, B.; Iwai, L. K.; Lima, I. F.; Cajado-Carvalho, D.; Portaro, F. C.V. 2017. In vitro cleavage of bioactive peptides by peptidases from Bothrops jararaca venom and its neutralization by bothropic antivenom produced by Butantan Institute: Major contribution of serine peptidases. Toxicon, 137, 114-119.

Machado, C. 2018. Acidentes ofídicos no Brasil: da assistência no município do Rio de Janeiro ao controle de saúde animal em instituto produtor de soro antiofídico. Tese de Doutorado, Instituto Oswaldo Cruz, Fundação Oswaldo Cruz. Rio de Janeiro, Rio de Janeiro, Brasil. 140p.

Maduwage, K.; Silva, A.; O’Leary, M. A.; Hodgson, W. C.; Isbister, G. K. 2016. Efficacy of Indian polyvalent snake antivenom against Sri Lankan snake venoms: lethality studies or clinically focussed in vitro studies. Sci. Rep., 6, 1-11.

Marques, O. A. V.; Sazima, I. 2004. História Natural dos répteis da Estação Ecológica Jureia-Itatins. In: Marques, O. A. V.; Duleba, W. Estação Ecológica Jureia-Itatins - ambiente físico, flora e fauna, Holos, pp. 257-277.

Matos, R. R.; Ignotti, E. 2020. Incidência de acidentes ofídicos por gêneros de serpentes nos biomas brasileiros. Ciênc. Saúde Colet., 25, (7), 2837-2846.

Melgarejo A. R. 2009. Serpentes peçonhentas do Brasil. In: Cardoso, J. L. C.; França, F. O. S., Wen, F. H.; Málaque, C. M. S.; Haddad Jr., V. Animais Peçonhentos no Brasil: Biologia, clínica e terapêutica dos acidentes. 2 ed., Savier, pp.42-70.

Navega-Gonçalves, M. E. C.; Porto, T. 2016. Conservação de serpentes nos biomas brasileiros. Bioikos, 30, (1), 55-76.

Pinho, F. M. O.; Pereira, I. D. 2001. Ofidismo. Rev. Ass. Med. Brasil., 47, (1), 24-29.
Salomão, M. G.; Oliveira-Luna, K. P.; Machado, C. 2018. Epidemiologia dos acidentes por animais peçonhentos e a distribuição de soros: estado de arte e a situação mundial. Rev. Salud Pública, 20, (4), 523-529.

Sanhajariya, S.; Duffull, S. B.; Isbister, G. K. 2018. Pharmacokinetics of snake venom. Toxins, 10, (73), 1-21.

Santana, C. R.; Oliveira, M. G. 2020. Avaliação do uso de soros antivenenos na emergência de um hospital público regional de Vitória da Conquista (BA), Brasil. Cienc. Saúde Coletiva, 25, (3), 869-878.

Silva, I. P. F.; Tarsitano, M. A. A.; Marchini, D. C.; Almeida, L. F. R. 2012. Expansão da cultura de cana-de-açúcar e sua caracterização no município de Birigui, Estado de São Paulo. Inf. Econômicas, SP, 42, (1), 54-65.

Silva, M. J. C.; Soares, F. T.; Trindade, G. P.; Diniz, H. S.; Medeiros, J. M. R.; Lopes, J. G. M.; Rocha, J. L. G.; Gonçalvez, L. O.; Nunes, P. C. 2019. Perfil epidemiológico dos acidentes ofídicos da mesorregião do baixo Amazonas do estado do Pará, Brasil. Braz. J. Hea. Rev., 2, (3), 1968-1979.

SINAN. Sistema de Informação de Agravos de Notificação. 2019. Disponível em: http://portalsinan.saude.gov.br/acidente-poranimais-peconhentos Acesso em: novembro de 2020.

Sitprija, V.; Sitprija, S. 2012. Renal effects and injury induced by animal toxins. Toxicon, 60, (5), 943-953.

Souza, R. C. G. 2009. Aspectos clínicos do acidente laquético. In: Cardoso, J. L. C.; França, F. O. S.; Wen, F. H.; Málaque, C. M. $\mathrm{S}$; Haddad Jr., V. Animais Peçonhentos no Brasil: biologia, clínica e terapêutica dos acidentes, 2 ed., Savier, pp. 96-107.

SVS $^{1}$. Secretaria de Vigilância em Saúde do Ministério da Saúde, Brasil. 2019. Vigilância Epidemiológica. In: Guia de Vigilância em Saúde, 3 ed., pp. 665-669. Disponível em: https://bvsms.saude.gov.br/bvs/publicacoes/g uia_vigilancia_saude_3ed.pdf. Acesso em: $12 / 2020$.

$\mathrm{SVS}^{2}$. Secretaria de Vigilância em Saúde do Ministério da Saúde, Brasil. 2019. Acidentes por animais peçonhentos. In: Guia de Vigilância em Saúde, 3 ed., pp. 652-655. Disponível em: https://bvsms.saude.gov.br/bvs/publicacoes/g uia_vigilancia_saude_3ed.pdf. Acesso em: $12 / 2020$.

Wen, F. H. 2009. Soroterapia. In: Cardoso, J. L. C.; França, F. O. S.; Wen, F. H.; Málaque, C. 
M. S.; Haddad Jr., V. Animais Peçonhentos no Brasil: biologia, clínica e terapêutica dos acidentes, 2 ed., Savier, pp. 432-445.

Wen, F. H.; Monteiro, W. M.; Silva, A. M. M.; Tambourgi, D. V.; Silva, I. M.; Sampaio, V. S.; Santos, M. C.; Sachett, J.; Ferreira, L. C. L.; Kalil, J.; Lacerda, M. 2015. Snakebites and scorpion stings in the Brazilian Amazon: identifying research priorities for a largely neglected for a largely neglected problem. PLoSNegl. Trop. Dis., 1-11.
Williams, D. J.; Faiz, M. A.; Abela-Ridder, B.; Ainsworth, S.; Bulfone, T. C.; Nickerson, A. D.; Habib. A. G.; Junghanss, T.; Fan, H. W.; Tumer, M.; Harrison, R. A.; Warrell, D. A. 2019. Strategy for a globally coordinated response to a priority neglected tropical disease: Snakebite envenoming. PLoS Negl. Trop. Dis., 13, (2), 1-12.

World Health Organization. Disponível em: https://www.who.int/activities/improvingecological-and-epidemiological-data-onsnakebite-envenoming. Acesso em: 12/2020. 